\title{
GAUSSIAN MEASURES OF DILATATIONS OF CONVEX SYMMETRIC SETS ${ }^{1}$
}

\author{
By RAFAŁ LATAŁA ${ }^{2}$ AND KRZYSZTOF OLESZKIEWICZ \\ Warsaw University
}

\begin{abstract}
We prove that the inequality $\Psi^{-1}(\mu(t A)) \geq t \Psi^{-1}(\mu(A))$ holds for any centered Gaussian measure $\mu$ on a separable Banach space $F$, any convex, closed, symmetric set $A \subset F$ and $t \geq 1$, where $\Psi(x)=\gamma_{1}(-x, x)=$ $(2 \pi)^{-1 / 2} \int_{-x}^{x} \exp \left(-y^{2} / 2\right) d y$. As an application, the best constants in comparison of moments of Gaussian vectors are calculated.
\end{abstract}

1. Main theorem. The main theorem we will prove in this paper is the following one previously known also as an $S$-conjecture.

THEOREM 1. Let $\mu$ be a centered Gaussian measure on a separable Banach space $F$. If $A$ is a symmetric, convex, closed subset of $F$ and $P \subset F$ is a symmetric strip, that is, $P=\left\{x \in F:\left|x^{*}(x)\right| \leq 1\right\}$ for some $x^{*} \in F^{*}$, such that $\mu(A)=\mu(P)$ then

$$
\mu(t A) \geq \mu(t P) \text { for } t \geq 1
$$

and

$$
\mu(t A) \leq \mu(t P) \quad \text { for } 0 \leq t \leq 1 .
$$

The question comes from an unpublished manuscript of L. A. Shepp (1969); later it was published by Szarek [6]. A simple approximation argument using representation of Gausssian measures, presented in detail in [4], shows that it is enough to prove Theorem 1 for $F=R^{n}$ and $\mu=\gamma_{n}$ - canonical Gaussian measure in $R^{n}$ (that is, the measure with density $(2 \pi)^{-n / 2} \exp \left(-|x|^{2} / 2\right)$ ). The positive answer for $n \leq 3$ was given by Sudakov and Zalgaller [5]. In the special case of $A$ in $R^{n}$ symmetric with respect to each coordinate, Theorem 1 was proved by Kwapień and Sawa [4].

Before formulating the next results, which will lead to the proof of Theorem 1 , let us state a few definitions. We will always assume below that $A$ is a subset of $R^{n}$ unless we state otherwise.

$$
\begin{aligned}
& \Phi(x)=\gamma_{1}(-\infty, x)=\frac{1}{\sqrt{2 \pi}} \int_{-\infty}^{x} \exp \left(-y^{2} / 2\right) d y, \\
& \Psi(x)=\gamma_{1}(-x, x)=\frac{1}{\sqrt{2 \pi}} \int_{-x}^{x} \exp \left(-y^{2} / 2\right) d y,
\end{aligned}
$$

Received October 1998; revised June 1999.

${ }^{1}$ Supported in part by Polish Grant KBN 2 PO3A 04315

${ }^{2}$ Supported in part by NSF Grant DMS-96-32032.

AMS 1991 subject classifications. Primary 60G15; secondary 60B11, 60E15.

Key words and phrases. Gaussian measures, moment inequalities, isoperimetry, convex bodies. 


$$
\begin{aligned}
A^{h} & =\left\{x \in R^{n}: \operatorname{dist}(x, A) \leq h\right\}-h \text {-neighborhood of } A, \\
\gamma_{n}^{+}(A) & =\liminf _{h \rightarrow 0+}\left(\gamma_{n}\left(A^{h}\right)-\gamma_{n}(A)\right) / h \text {-Gaussian perimeter of } A, \\
w(A) & =\sup \{r: B(0, r) \subset A\} .
\end{aligned}
$$

Let us note that for a symmetric strip $P, w(P)$ is equal to half of the width of $P$ and for a symmetric convex set $A$,

$$
w(A)=\inf \left\{w(P): A \subset P, P \text { is a symmetric strip in } R^{n}\right\} .
$$

Thus $2 w(A)$ can be considered as the width of the set $A$.

THEOREM 2. $\quad$ Suppose that $\gamma_{2}(A)=\gamma_{2}(P)$ where $P$ is a symmetric strip with width $2 p$ and $A$ is a set in $R^{2}$ symmetric about the $y$-axis, lying under the graph of some symmetric, smooth, concave function $f:(-w, w) \rightarrow R$, nonincreasing on $[0, w)$ with $\lim _{x \rightarrow w-} f(x)=-\infty$. Then

$$
w \gamma_{2}^{+}(A) \geq w(P) \gamma_{2}^{+}(P)=\sqrt{\frac{2}{\pi}} p \exp \left(\frac{-p^{2}}{2}\right) .
$$

We postpone the proof of Theorem 2 till the end of the paper and now show how it implies the main result and the following theorem.

THEOREM 3. If $\gamma_{n}(A)=\gamma_{n}(P)$ where $P$ is a symmetric strip and $A$ is a convex symmetric set in $R^{n}$, then

$$
w(A) \gamma_{n}^{+}(A) \geq w(P) \gamma_{n}^{+}(P) .
$$

Proof. For $n=1$ there is nothing to prove, so we will assume that $n \geq 2$. Let $w=w(A)$; without loss of generality we may then assume that

$$
A \subset\left\{x \in R^{n}:\left|x_{1}\right| \leq w\right\} .
$$

For $x \in(-w, w)$ let

$$
A_{x}=\left\{y \in R^{n-1}:(x, y) \in A\right\}
$$

and

$$
f(x)=\Phi^{-1}\left(\gamma_{n-1}\left(A_{x}\right)\right) .
$$

Then by the convexity of $A$ and Ehrhard's inequality [1] $f$ is concave; moreover, it is symmetric and hence nonincreasing on $[0, w)$. Let us define

$$
B=\left\{(x, y) \in R^{2}:|x|<w, y \leq f(x)\right\} ;
$$

thus we have $\gamma_{2}(B)=\gamma_{n}(A)=\gamma_{n}(P)$. Let $h>0, x \in(-w-h, w+h)$ and $y \in$ $\left(B^{h}\right)_{x}$; then there exists a point $\left(x^{\prime}, y^{\prime}\right) \in B$ such that $\left|x-x^{\prime}\right|=h_{1},\left|y-y^{\prime}\right|=h_{2}$ and $h_{1}^{2}+h_{2}^{2} \leq h^{2}$. Since $\left(A_{x^{\prime}}\right)^{h_{2}} \subset\left(A^{h}\right)_{x}$ we get by the isoperimetric inequality

$$
\begin{aligned}
\Phi^{-1}\left(\gamma_{n-1}\left(\left(A^{h}\right)_{x}\right)\right) & \geq \Phi^{-1}\left(\gamma_{n-1}\left(\left(A_{x^{\prime}}\right)^{h_{2}}\right)\right) \\
& \geq \Phi^{-1}\left(\gamma_{n-1}\left(A_{x^{\prime}}\right)\right)+h_{2} \geq y^{\prime}+h_{2} \geq y .
\end{aligned}
$$


Taking the supremum over all $y \in\left(B^{h}\right)_{x}$, we get that $\gamma_{1}\left(\left(B^{h}\right)_{x}\right) \leq \gamma_{n-1}\left(\left(A^{h}\right)_{x}\right)$ for any $h>0$ and $x \in(-w-h, w+h)$. Thus $\gamma_{2}\left(B^{h}\right) \leq \gamma_{n}\left(A^{h}\right)$ and $\gamma_{2}^{+}(B) \leq$ $\gamma_{n}^{+}(A)$. Therefore it is enough to prove that

$$
w \gamma_{2}^{+}(B) \geq w(P) \gamma_{n}^{+}(P) .
$$

An easy approximation argument shows that we may assume that $f$ is smooth and $\lim _{x \rightarrow w-} f(x)=-\infty$, so (1) follows by Theorem 2 .

Proof of Theorem 1. Let us define for any measurable set $B$ in $R^{n}$,

$$
\gamma_{B}(t)=\gamma_{n}(t B) \text { for } t>0 \text {. }
$$

Taking derivatives of both sides of inequalities in Theorem 1 (for details see [4]), one can see that it is enough to show that for any convex closed symmetric set $A$ in $R^{n}$ we have

$$
\gamma_{A}^{\prime}(1) \geq \gamma_{P}^{\prime}(1)
$$

where $P$ is a strip $P=\left\{\left|x_{1}\right| \leq p\right\}$ such that $\gamma_{n}(A)=\gamma_{n}(P)$. Let $w=w(A)$, so $B(0, w) \subset A$. Let us notice that for $t>1$ if $x \in A$ then $B\left(t^{-1} x,(t-1) w / t\right)=$ $t^{-1} x+\left(1-t^{-1}\right) B(0, w) \subset A$ so $B(x,(t-1) w) \subset t A$, hence

$$
A^{(t-1) w} \subset t A \text {. }
$$

Therefore

$$
\gamma_{A}^{\prime}(1) \geq w \gamma_{n}^{+}(A)=w(A) \gamma_{n}^{+}(A) .
$$

Moreover, for the strip $P$,

$$
\gamma_{P}^{\prime}(1)=\sqrt{\frac{2}{\pi}} p \exp \left(\frac{-p^{2}}{2}\right)=w(P) \gamma_{n}^{+}(P)
$$

and (2) follows by Theorem 3 .

The following corollary is just a reformulation of Theorem 1 . The second part of it was proved in [2].

COROLlaRY 1. If $\mu$ is a centered Gaussian measure on a separable Banach space $F$ and $B$ is a convex, symmetric, closed subset of $F$, then

$$
\mu(r B) \geq \Psi\left(\frac{r}{s} \Psi^{-1}(\mu(s B))\right) \quad \text { for } r \geq s>0 .
$$

In particular for each $b<1$ there exists a constant $C_{b}<\infty$ depending only on $b$ such that if $\mu(B) \leq b$, then

$$
\mu(t B) \leq C_{b} t \mu(B) \quad \text { for } t \in[0,1] .
$$

The next corollary can be considered as some kind of isoperimetric inequality for convex, symmetric sets. 
Corollary 2. For any convex, symmetric subset A of a symmetric strip $P$ in $R^{n}$ and any $h>0$, the following inequality holds:

$$
\frac{\Psi^{-1}\left(\gamma_{n}\left(A^{h}\right)\right)}{\Psi^{-1}\left(\gamma_{n}(A)\right)} \geq \frac{\Psi^{-1}\left(\gamma_{n}\left(P^{h}\right)\right)}{\Psi^{-1}\left(\gamma_{n}(P)\right)} \text {. }
$$

Proof. Notice that $w\left(A^{h}\right)=w(A)+h$. Consider the function $r(h)=$ $\Psi^{-1}\left(\gamma_{n}\left(A^{h}\right)\right) / w\left(A^{h}\right)$. From the definition of $\gamma_{n}^{+}$we deduce that

$$
\begin{aligned}
& \liminf _{\varepsilon \rightarrow 0^{+}} \frac{r(h+\varepsilon)-r(h)}{\varepsilon} \\
& \quad=\frac{1}{w\left(A^{h}\right)^{2}}\left(\sqrt{\frac{\pi}{2}} \gamma_{n}^{+}\left(A^{h}\right) w\left(A^{h}\right) \exp \left(\Psi^{-1}\left(\gamma_{n}\left(A^{h}\right)\right)^{2} / 2\right)-\Psi^{-1}\left(\gamma_{n}\left(A^{h}\right)\right)\right) \geq 0,
\end{aligned}
$$

by Theorem 3 applied to the set $A^{h}$.

The function $r(h)$ is continuous, hence $r(h) \geq r(0)$ for any $h>0$. Therefore,

$$
\begin{aligned}
\Psi^{-1}\left(\gamma_{n}\left(A^{h}\right)\right) & =\left(w\left(A^{h}\right)\right) r(h) \geq(w(A)+h) r(0)=\Psi^{-1}\left(\gamma_{n}(A)\right)\left(1+\frac{h}{w(A)}\right) \\
& \geq \Psi^{-1}\left(\gamma_{n}(A)\right)\left(1+\frac{h}{w(P)}\right)=\Psi^{-1}\left(\gamma_{n}(A)\right) \frac{\Psi^{-1}\left(\gamma_{n}\left(P^{h}\right)\right)}{\Psi^{-1}\left(\gamma_{n}(P)\right)},
\end{aligned}
$$

which completes the proof.

Finally, as a consequence of Theorem 1 let us state the following result which gives the best constants in comparison of moments of Gaussian vectors. The proof presented below is due to S. Szarek (private communication).

COROLlARY 3. If $g_{i}$ are independent standard normal r.v. and $x_{i}$ are vectors in some separable Banach space $(E,\|\cdot\|)$ such that the series $S=\sum x_{i} g_{i}$ is a.s. convergent, then

$$
\left(E\|S\|^{p}\right)^{1 / p} \leq \frac{\gamma_{p}}{\gamma_{q}}\left(E\|S\|^{q}\right)^{1 / q} \quad \text { for any } p \geq q>0,
$$

where

$$
\gamma_{p}=\left(E\left|g_{1}\right|^{p}\right)^{1 / p}=\sqrt{2}\left(\frac{1}{\sqrt{\pi}} \Gamma\left(\frac{p+1}{2}\right)\right)^{1 / p} .
$$

Proof. Let $a \in R$ be such that $E\|S\|^{p}=E\left|a g_{1}\right|^{p}$. Then

$$
\int_{0}^{\infty} t^{p-1} P(\|S\|>t) d t=\int_{0}^{\infty} t^{p-1} P\left(\left|a g_{1}\right|>t\right) d t
$$

So for some $t_{0}>0$ we have $P\left(\|S\|>t_{0}\right)=P\left(\left|a g_{1}\right|>t_{0}\right)$. Applying Theorem 1 we easily obtain that $P(\|S\|>t) \geq P\left(\left|a g_{1}\right|>t\right)$ for $0 \leq t \leq t_{0}$ and $P(\|S\|>$ $t) \leq P\left(\left|a g_{1}\right|>t\right)$ for $t \geq t_{0}$. Therefore for $t>0$ and $p \geq q>0$, we get

$$
\left(\frac{t}{t_{0}}\right)^{p-1}\left(P(\|S\|>t)-P\left(\left|a g_{1}\right|>t\right)\right) \leq\left(\frac{t}{t_{0}}\right)^{q-1}\left(P(\|S\|>t)-P\left(\left|a g_{1}\right|>t\right)\right) \text {. }
$$


This gives

$$
\int_{0}^{\infty} t^{q-1} P(\|S\|>t) d t \geq \int_{0}^{\infty} t^{q-1} P\left(\left|a g_{1}\right|>t\right) d t
$$

that is, $E\|S\|^{q} \geq E\left|a g_{1}\right|^{q}$ and proves (3).

Proof of Theorem 2. During this section we will frequently use the following functions

$$
\begin{aligned}
& T(y)=1-\Phi(y), \\
& h(y)=2 \pi T(y)^{2} \exp \left(y^{2}\right) .
\end{aligned}
$$

LEMMA 1. The function $h(y)$ is decreasing for $y \geq 0$.

PROOF. We have to prove that $T(y) \exp \left(y^{2} / 2\right)$ is a decreasing function of $y$ on $(0, \infty)$. To see this note that

$$
\begin{aligned}
\frac{d}{d y}\left(T(y) \exp \left(\frac{y^{2}}{2}\right)\right) & =\frac{1}{\sqrt{2 \pi}}\left(y \exp \left(\frac{y^{2}}{2}\right) \int_{y}^{\infty} \exp \left(\frac{-s^{2}}{2}\right) d s-1\right) \\
& <\frac{1}{\sqrt{2 \pi}}\left(\exp \left(\frac{y^{2}}{2}\right) \int_{y}^{\infty} s \exp \left(\frac{-s^{2}}{2}\right) d s-1\right)=0 .
\end{aligned}
$$

LEMMA 2. The function $g(y)=h(y)^{-1}-y^{2}$ is nondecreasing for $y \geq 0$, in particular,

$$
h(y)^{-1} \geq y^{2}+1.5 \text { for } y>1.5
$$

and

$$
\sqrt{2 \pi} T(y) \geq \frac{1}{\sqrt{y^{2}+2}} \exp \left(\frac{-y^{2}}{2}\right) \text { for } y>0 \text {. }
$$

ProOF. First let us notice that the function $\varphi(y)=\sqrt{2 \pi} T(y)-\exp \left(-y^{2} / 2\right) /$ $\sqrt{y^{2}+2}$ is decreasing on $(0, \infty)$. Indeed,

$$
\begin{aligned}
\left(y^{2}+2\right)^{3 / 2} \exp \left(\frac{y^{2}}{2}\right) \varphi^{\prime}(y) & =y^{3}+3 y-\left(y^{2}+2\right)^{3 / 2} \\
& =\frac{1}{y^{3}+3 y+\left(y^{2}+2\right)^{3 / 2}}\left(\left(y^{3}+3 y\right)^{2}-\left(y^{2}+2\right)^{3}\right) \\
& =-\frac{3 y^{2}+8}{y^{3}+3 y+\left(y^{2}+2\right)^{3 / 2}}<0 .
\end{aligned}
$$

As $\lim _{y \rightarrow \infty} \varphi(y)=0$, we obtain the inequality (4). We have also

$$
\begin{aligned}
T(y) & =\frac{1}{\sqrt{2 \pi}} \int_{y}^{\infty} \exp \left(\frac{-s^{2}}{2}\right) d s \leq \frac{1}{\sqrt{2 \pi} y} \int_{y}^{\infty} s \exp \left(\frac{-s^{2}}{2}\right) d s \\
& =\frac{1}{\sqrt{2 \pi}} \frac{\exp \left(-y^{2} / 2\right)}{y} .
\end{aligned}
$$


By (4) and (5) we deduce that $0 \leq g(y) \leq 2$ for $y \geq 0$. Fix $a \in[0,2]$. We only need to prove that if $g\left(y_{a}\right) \geq a$ for some $y_{a}>0$ then also $g(y) \geq a$ for all $y \geq$ $y_{a}$. Now, $g(y) \geq a$ is equivalent to $T(y) \leq 1 / \sqrt{2 \pi}\left(\exp \left(-y^{2} / 2\right) / \sqrt{y^{2}+a}\right)$. Let us investigate behavior of the function $\psi_{a}(y)=1 / \sqrt{2 \pi} \exp \left(-y^{2} / 2\right) / \sqrt{y^{2}+a}-$ $T(y)$. We have

$$
\sqrt{2 \pi} \exp \left(y^{2} / 2\right)\left(y^{2}+a\right)^{3 / 2} \psi_{a}^{\prime}(y)=\left(y^{2}+a\right)^{3 / 2}-y-y\left(y^{2}+a\right) .
$$

Hence $\psi_{a}^{\prime}(y) \geq 0$ if and only if $\left(y^{2}+a\right)^{3} \geq\left(y^{3}+(a+1) y\right)^{2}$, which is equivalent to $(2-a) y^{4}+\left(1+2 a-2 a^{2}\right) y^{2}-a^{3} \leq 0$. The left-hand side of the last inequality is a second-degree polynomial in $y^{2}$ with nonnegative leading coefficient $2-a$. Moreover, for $y=0$ the last inequality is obviously satisfied. Therefore there exists a nonnegative number $m_{a}$ such that $\psi_{a}$ is nondecreasing on the interval $\left(0, m_{a}\right)$ and it is nonincreasing on the interval $\left(m_{a}, \infty\right) \cdot \operatorname{As} \lim _{y \rightarrow \infty} \psi_{a}(y)=0$, this proves that $\psi_{a}\left(y_{a}\right) \geq 0$ implies $\psi_{a}(y) \geq 0$ for all $y \geq y_{a}$, which completes the proof, since $h(1.5)^{-1} \geq 1.5^{2}+1.5$.

LEMMA 3. The function $x T(x) \exp \left(x^{2} / 2\right)$ is increasing on $[0, \infty)$.

Proof. We have

$$
\left(\sqrt{2 \pi} x T(x) \exp \left(x^{2} / 2\right)\right)^{\prime}=\left(1+x^{2}\right) \sqrt{2 \pi} T(x) \exp \left(x^{2} / 2\right)-x
$$

so it is enough to show that $\sqrt{h(x)}>x /\left(x^{2}+1\right)$. But by Lemma 2 ,

$$
\sqrt{h(x)} \geq \frac{1}{\sqrt{x^{2}+2}}>\frac{x}{x^{2}+1}
$$

LEMMA 4. The function $F(x)=h(x)^{-1}+2 \ln T(x)$ is nonincreasing on $[0, \infty)$.

Proof. First let us note that, due to a well-known Komatsu's estimate (see [3], page 17),

$$
T(x) \geq \frac{1}{\sqrt{2 \pi}} \frac{2}{x+\sqrt{x^{2}+4}} \exp \left(\frac{-x^{2}}{2}\right)
$$

Hence $\sqrt{h(x)} \geq 2 /\left(x+\sqrt{x^{2}+4}\right)$ and therefore

$$
x \sqrt{h(x)} \geq \frac{2 x}{x+\sqrt{x^{2}+4}}=1-\left(\frac{2}{x+\sqrt{x^{2}+4}}\right)^{2} \geq 1-h(x) .
$$


So,

$$
\begin{aligned}
F^{\prime}(x) & =-\frac{h^{\prime}(x)}{h(x)^{2}}+2 \frac{T^{\prime}(x)}{T(x)} \\
& =-\frac{1}{h(x)} \frac{\left(2 \pi T(x)^{2} \exp \left(x^{2}\right)\right)^{\prime}}{2 \pi T(x)^{2} \exp \left(x^{2}\right)}-\frac{2 \exp \left(-x^{2} / 2\right)}{\sqrt{2 \pi} T(x)} \\
& =-\frac{2}{h(x)}\left(\frac{T^{\prime}(x)}{T(x)}+x\right)-\frac{2}{\sqrt{h(x)}}=\frac{2}{h(x)^{3 / 2}}(1-h(x)-x \sqrt{h(x)}) \leq 0
\end{aligned}
$$

and the proof is complete.

LEMMA 5. For any real $y$ we have $\Phi^{2}(y) h(y) \leq \pi / 8$.

PROOF. Note that

$$
\begin{aligned}
& \frac{8}{\pi} \Phi(y)^{2} h(y) \exp \left(-y^{2}\right) \\
& \quad=(4 \Phi(y) T(y))^{2} \\
& =\left(1-\gamma_{2}([-|y|,|y|] \times[-|y|,|y|])\right)^{2} \leq\left(1-\gamma_{2}\left(B_{2}(0,|y|)\right)\right)^{2} \\
& \quad=\left(1-\frac{1}{2 \pi} \int_{0}^{|y|} \exp \left(\frac{-r^{2}}{2}\right) 2 \pi r d r\right)^{2}=\left(\exp \left(\frac{-y^{2}}{2}\right)\right)^{2}=\exp \left(-y^{2}\right)
\end{aligned}
$$

and the proof is complete.

LEMMA 6. Let $f$ be a nonincreasing integrable function on $(0, \infty)$ and $\mu$ any finite positive measure on $(0, \infty)$. Then for any $0 \leq a_{1}<b_{1} \leq \infty$, $0 \leq a_{2}<b_{2} \leq \infty$ such that $a_{1} \leq a_{2}$ and $b_{1} \leq b_{2}$ we have

$$
\frac{\int_{a_{1}}^{b_{1}} f(x) d \mu(x)}{\mu\left(a_{1}, b_{1}\right)} \geq \frac{\int_{a_{2}}^{b_{2}} f(x) d \mu(x)}{\mu\left(a_{2}, b_{2}\right)} .
$$

The proof is obvious.

LEMMA 7. For any $0 \leq c_{1}<d_{1} \leq \infty, 0 \leq c_{2}<d_{2} \leq \infty$ such that $c_{1} \leq c_{2}$ and $d_{1} \leq d_{2}$ we have

$$
\frac{\Phi\left(d_{1}\right)-\Phi\left(c_{1}\right)}{\exp \left(-c_{1}^{2} / 2\right)-\exp \left(-d_{1}^{2} / 2\right)} \geq \frac{\Phi\left(d_{2}\right)-\Phi\left(c_{2}\right)}{\exp \left(-c_{2}^{2} / 2\right)-\exp \left(-d_{2}^{2} / 2\right)}
$$

ProOF. Let us notice that

$$
\sqrt{2 \pi}(\Phi(d)-\Phi(c))=\int_{c^{2}}^{d^{2}} \frac{1}{2 \sqrt{y}} \exp \left(\frac{-y}{2}\right) d y
$$

and we may apply Lemma 6 with $f(y)=1 / \sqrt{y}$. 
LEMMA 8. Suppose that $s \geq u>0$ and $p>0$ satisfy the inequality

$$
1-\Phi(u) \leq 1-\Phi(p)+\frac{1}{2}(1-\Phi(s)),
$$

then

$$
\frac{1}{2} \exp \left(\left(u^{2}-s^{2}\right) / 2\right)+\exp \left(\left(u^{2}-p^{2}\right) / 2\right) \geq 1 .
$$

Proof. If $u \geq p$ then (7) is obvious so we may assume that $p>u$. Inequality (6) immediately implies that

$$
\frac{\Phi(p)-\Phi(u)}{1-\Phi(s)} \leq \frac{1}{2}
$$

and by Lemma 7,

$$
\frac{\Phi(p)-\Phi(u)}{1-\Phi(s)} \geq \frac{\exp \left(-u^{2} / 2\right)-\exp \left(-p^{2} / 2\right)}{\exp \left(-s^{2} / 2\right)} .
$$

From the above two inequalities, (7) immediately follows.

LEMMA 9. If $c>0$ and $p_{0}>0$ are such that $\exp \left(-c p_{0}\right) \leq 1-p_{0}$ then $\exp (-c p) \leq 1-p$ for all $p \in\left[0, p_{0}\right]$. In particular,

$$
(1-p) \exp (4 p / \pi) \geq 1 \text { for all } p \in[0,1 / 3]
$$

and

$$
(1-p) \exp (4 p /(\pi-4 / 9)) \geq 1 \text { for all } p \in[0,1 / 2]
$$

Proof. The function $\exp (-c p)-1+p$ is a convex function of $p$ and that implies first part. The last statements follow by the first one and inequalities $\exp (4 / 3 \pi) \geq 3 / 2$ and $\exp (2 /(\pi-4 / 9)) \geq 2$.

LEMMA 10. For any $p \in(0,1 / 2]$ and $z \geq 0$,

$$
p \exp \left(-\pi z^{2} / 16 p^{2}\right)+(1-p) \geq \exp (-z / 2) .
$$

Proof. Using Taylor's expansion, we have

$$
\begin{aligned}
p \exp & \left(\frac{z}{2}-\frac{\pi z^{2}}{16 p^{2}}\right)+(1-p) \exp \left(\frac{z}{2}\right) \\
\geq & p\left(1+\frac{z}{2}-\frac{\pi}{16 p^{2}} z^{2}\right)+(1-p)\left(1+\frac{z}{2}+\frac{z^{2}}{8}\right) \\
& =1+\frac{z}{2}-\frac{\pi+2(p-1) p}{16 p} z^{2},
\end{aligned}
$$

so inequality is satisfied for $z \leq 8 p /(\pi+2(p-1) p)$. It is enough to show that

$$
f(p)=(1-p) \exp (4 p /(\pi+2(p-1) p)) \geq 1 \text {. }
$$


If $p \leq 1 / 3$ then $f(p) \geq(1-p) \exp (4 p / \pi) \geq 1$ by the previous lemma. If $p \in[1 / 3,1 / 2]$, then $(1-p) p \geq 2 / 9$, so again,

$$
f(p) \geq(1-p) \exp (4 p /(\pi-4 / 9)) \geq 1 \text {. }
$$

LEMMA 11. If $y \leq 1.5$ and $z \geq 0$ or if $0 \leq z \leq y^{2}+1.5$, then

$$
\Phi(y) \exp \left(-h(y) z^{2} / 2\right)+1-\Phi(y) \geq \exp (-z / 2) \text {. }
$$

Proof. If $y \leq 0$ then the lemma follows by Lemmas 10 and 5. For $y>0$, put $R_{y}(z)=\exp (-z / 2)-\Phi(y) \exp \left(-h(y) z^{2} / 2\right)$ and $M(y)=\sup _{z>1 / h(y)} R_{y}(z)$. First note that in view of Lemma $1, R_{y}(z)$ is a decreasing function of positive argument $y$ for any fixed $z$. As $1 / h(y)$ is an increasing function for $y>0$, we see that $\sup _{z>1 / h(y)}$ is taken over a decreasing set. Together these facts show that $M(y)$ is nonincreasing for $y>0$. We have

$$
\frac{\partial}{\partial z} R_{y}(z)=R_{y}^{\prime}(z)=-\frac{1}{2} \exp \left(\frac{-z}{2}\right)+\Phi(y) h(y) z \exp \left(\frac{-h(y) z^{2}}{2}\right) .
$$

Therefore $R_{y}^{\prime}(0)<0$ and $R_{y}^{\prime}(z)<0$ for $z$ large enough. Note that $R_{y}^{\prime}(1 / h(y))=$ $(\Phi(y)-1 / 2) \exp \left(-1 /(2 h(y))>0\right.$. As $R_{y}^{\prime}(z)=, 0$ if and only if $\ln (2 \Phi(y) h(y) z)=$ $h(y) z^{2} / 2-z / 2$ we deduce that the function $R_{y}$ has for each fixed $y>0$ at most two local extrema on $(0, \infty)$ because the left-hand side of the last equation is concave and the right-hand side is convex. Summarizing these facts we arrive at the conclusion that for each $y>0$ there exist positive numbers $\alpha(y)<\beta(y)$ such that the function $R_{y}$ is decreasing on the interval $(0, \alpha(y))$, increasing on the interval $(\alpha(y), \beta(y))$ to which $1 / h(y)$ belongs and again decreasing on the interval $(\beta(y), \infty)$. Therefore, to prove our main claim, that is, that $T(y) \geq R_{y}(z)$ for any $y \in[0,1.5], z \geq 0$, it is enough to prove that $T(y) \geq M(y)$, as in the points $z=0$ and $z=1 / h(y)$ the claim is trivial.

Let us consider Table 1 . In the $k$ th row, $T_{1}$ should be understood as $T_{1}\left(y_{k}\right)$;

\begin{tabular}{|c|c|c|c|c|c|c|c|c|c|c|c|c|}
\hline $\boldsymbol{k}$ & $\boldsymbol{y}_{\boldsymbol{k}}$ & $T_{1}$ & $T_{2}$ & $\Phi_{1}$ & $\Phi_{2}$ & $h_{1}$ & $h_{2}$ & $z_{k}$ & $Z_{k}$ & $a_{k}$ & $\boldsymbol{b}_{\boldsymbol{k}}$ & $M_{k}$ \\
\hline 1 & 0.00 & 0.500 & 0.500 & 0.500 & 0.500 & 1.570 & 1.571 & 1.34 & 1.35 & 0.256 & 0.254 & 0.393 \\
\hline 2 & 0.25 & 0.401 & 0.402 & 0.598 & 0.599 & 1.075 & 1.081 & 1.78 & 1.81 & 0.206 & 0.202 & 0.309 \\
\hline 3 & 0.49 & 0.311 & 0.313 & 0.687 & 0.689 & 0.772 & 0.783 & 2.26 & 2.33 & 0.162 & 0.155 & 0.242 \\
\hline 4 & 0.69 & 0.244 & 0.246 & 0.754 & 0.756 & 0.602 & 0.613 & 2.70 & 2.80 & 0.130 & 0.123 & 0.192 \\
\hline 5 & 0.87 & 0.192 & 0.193 & 0.807 & 0.808 & 0.493 & 0.499 & 3.16 & 3.25 & 0.104 & 0.098 & 0.149 \\
\hline 6 & 1.04 & 0.149 & 0.150 & 0.850 & 0.851 & 0.411 & 0.417 & 3.62 & 3.72 & 0.082 & 0.077 & 0.117 \\
\hline 7 & 1.18 & 0.118 & 0.120 & 0.880 & 0.882 & 0.351 & 0.365 & 3.97 & 4.22 & 0.069 & 0.060 & 0.104 \\
\hline 8 & 1.25 & 0.105 & 0.106 & 0.894 & 0.895 & 0.330 & 0.337 & 4.23 & 4.40 & 0.061 & 0.055 & 0.087 \\
\hline 9 & 1.35 & 0.088 & 0.089 & 0.911 & 0.912 & 0.300 & 0.308 & 4.53 & 4.72 & 0.052 & 0.047 & 0.075 \\
\hline 10 & 1.43 & 0.076 & 0.077 & 0.923 & 0.924 & 0.280 & 0.288 & 4.76 & 4.99 & 0.047 & 0.041 & 0.067 \\
\hline 11 & 1.49 & 0.068 & 0.069 & 0.931 & 0.932 & 0.267 & 0.276 & 4.92 & 5.20 & 0.043 & 0.037 & 0.064 \\
\hline 12 & 1.52 & 0.064 & & & & & & & & & & \\
\hline
\end{tabular}
one should understand the five next columns similarly.

TABLE 1 
We leave to the reader to check that for $k=1, \ldots, 11$ the numbers in the table satisfy the following inequalities:

$$
\begin{aligned}
T_{1}\left(y_{k}\right) \leq & T\left(y_{k}\right) \leq T_{2}\left(y_{k}\right), \quad \Phi_{1}\left(y_{k}\right) \leq \Phi\left(y_{k}\right) \leq \Phi_{2}\left(y_{k}\right), \\
h_{1}\left(y_{k}\right) \leq & 2 \pi T_{1}\left(y_{k}\right)^{2} \exp \left(y_{k}^{2}\right) \leq h\left(y_{k}\right), \\
h_{2}\left(y_{k}\right) \geq & 2 \pi T_{2}\left(y_{k}\right)^{2} \exp \left(y_{k}^{2}\right) \geq h\left(y_{k}\right), \\
z_{k} \leq & Z_{k}, \quad \frac{1}{2} \exp \left(-z_{k} / 2\right) \leq a_{k}, \quad \frac{1}{2} \exp \left(-Z_{k} / 2\right) \geq b_{k}, \\
& \Phi_{1}\left(y_{k}\right) h_{1}\left(y_{k}\right) z_{k} \exp \left(-h_{2}\left(y_{k}\right) z_{k}^{2} / 2\right) \geq a_{k}, \\
& \Phi_{2}\left(y_{k}\right) h_{2}\left(y_{k}\right) Z_{k} \exp \left(-h_{1}\left(y_{k}\right) Z_{k}^{2} / 2\right) \leq b_{k}
\end{aligned}
$$

and

$$
T_{1}\left(y_{k+1}\right) \geq M_{k} \geq \exp \left(-z_{k} / 2\right)-\Phi_{1}\left(y_{k}\right) \exp \left(-h_{2}\left(y_{k}\right) Z_{k}^{2} / 2\right) .
$$

Note also that $T_{1}\left(y_{12}\right) \leq T\left(y_{12}\right)$.

Now we are in a position to prove our claim. For each $y \in[0,1.5]$ we can find $k \in\{1, \ldots, 11\}$ such that $y_{k} \leq y \leq y_{k+1}$. Note that

$$
\begin{aligned}
R_{y_{k}}^{\prime}\left(z_{k}\right) & =-\frac{1}{2} \exp \left(-z_{k} / 2\right)+\Phi\left(y_{k}\right) h\left(y_{k}\right) z_{k} \exp \left(-h\left(y_{k}\right) z_{k}^{2} / 2\right) \\
& \geq-\frac{1}{2} \exp \left(-z_{k} / 2\right)+\Phi_{1}\left(y_{k}\right) h_{1}\left(y_{k}\right) z_{k} \exp \left(-h_{2}\left(y_{k}\right) z_{k}^{2} / 2\right) \\
& \geq-a_{k}+a_{k}=0,
\end{aligned}
$$

while

$$
\begin{aligned}
R_{y_{k}}^{\prime}\left(Z_{k}\right) & =-\frac{1}{2} \exp \left(-Z_{k} / 2\right)+\Phi\left(y_{k}\right) h\left(y_{k}\right) Z_{k} \exp \left(-h\left(y_{k}\right) Z_{k}^{2} / 2\right) \\
& \leq-\frac{1}{2} \exp \left(-Z_{k} / 2\right)+\Phi_{2}\left(y_{k}\right) h_{2}\left(y_{k}\right) Z_{k} \exp \left(-h_{1}\left(y_{k}\right) Z_{k}^{2} / 2\right) \\
& \leq-b_{k}+b_{k}=0,
\end{aligned}
$$

which means that $z_{k} \leq \beta_{k}=\beta\left(y_{k}\right) \leq Z_{k}$. Therefore,

$$
\begin{aligned}
M(y) & \leq M\left(y_{k}\right)=R_{y_{k}}\left(\beta_{k}\right)=\exp \left(-\beta_{k} / 2\right)-\Phi\left(y_{k}\right) \exp \left(-h\left(y_{k}\right) \beta_{k}^{2} / 2\right) \\
& \leq \exp \left(-z_{k} / 2\right)-\Phi_{1}\left(y_{k}\right) \exp \left(-h_{2}\left(y_{k}\right) Z_{k}^{2} / 2\right) \leq M_{k} \\
& \leq T_{1}\left(y_{k+1}\right) \leq T\left(y_{k+1}\right) \leq T(y),
\end{aligned}
$$

which completes the proof in the case of $y<1.5$. If $y \geq 1.5$, notice that we have already proved (8) for $0 \leq z \leq 1 / h(y)$. Thus Lemma 2 implies (8) for $0 \leq z \leq y^{2}+1.5$.

LEMMA 12. Let $w \geq a \geq x \geq 0$ and $y \in R$ satisfy the inequality

$$
\Phi(y) \Phi(w)+(1-\Phi(y)) \Phi(x) \geq \Phi(a) .
$$

Then if $y \leq 1.5$ or $a^{2}-x^{2} \leq y^{2}+1.5$ we have

$$
\begin{aligned}
w \sqrt{1+k^{2}} \exp \left(-y^{2} / 2\right) \geq & \sqrt{2 \pi}\left(a^{2}-x^{2}\right)(1-\Phi(y)) \\
& +k x \exp \left(-y^{2} / 2\right) \quad \text { for any } k \geq 0 .
\end{aligned}
$$


PROOF. Dividing both sides of (10) by $\sqrt{1+k^{2}}$ and taking the supremum over $k$, we have to prove that

$$
w^{2} \geq h(y) z^{2}+x^{2}
$$

where $z=a^{2}-x^{2}$. Suppose that this is not true, then by (9) we get that

$$
\Phi(y) \Phi\left(\sqrt{h(y) z^{2}+x^{2}}\right)+(1-\Phi(y)) \Phi(x)>\Phi(a)
$$

so

$$
\Phi(y)\left(\Phi\left(\sqrt{h(y) z^{2}+x^{2}}\right)-\Phi(a)\right)>(1-\Phi(y))(\Phi(a)-\Phi(x)) .
$$

Hence obviously $h(y) z^{2}+x^{2}>a^{2}$. Let us notice that

$$
\begin{aligned}
\sqrt{2 \pi}(\Phi(a)-\Phi(x)) & =\int_{x^{2}}^{a^{2}} \frac{1}{2 \sqrt{y}} \exp (-y / 2) d y \\
& \geq \frac{1}{a}\left(\exp \left(\frac{-x^{2}}{2}\right)-\exp \left(\frac{-a^{2}}{2}\right)\right) \\
& =\frac{1}{a} \exp \left(-x^{2} / 2\right)(1-\exp (-z / 2)) .
\end{aligned}
$$

In a similar way we show that

$$
\begin{aligned}
& \sqrt{2 \pi}\left(\Phi\left(\sqrt{h(y) z^{2}+x^{2}}\right)-\Phi(a)\right) \\
& \quad \leq \frac{1}{a} \exp \left(\frac{-x^{2}}{2}\right)\left(\exp \left(\frac{-z}{2}\right)-\exp \left(-h(y) z^{2} / 2\right)\right) .
\end{aligned}
$$

By (11), (12) and (13) we obtain

$$
\exp \left(\frac{-z}{2}\right)>\Phi(y) \exp \left(-h(y) z^{2} / 2\right)+1-\Phi(y),
$$

which contradicts Lemma 11.

LEMMA 13. If $p>0$ and $q$ satisfy the condition

$$
\frac{1}{2}(1-\Phi(q))=1-\Phi(p)
$$

then

$$
4 \exp \left(q^{2}-p^{2}\right) p^{2}-p^{2} \leq \ln 4
$$

Proof. Note that $q<p$. We will consider several cases 
CASE 1. $q^{2}>p^{2}$. Then $-q>p$ and therefore $1-\Phi(q)=\Phi(-q) \geq \Phi(p)=$ $\frac{1}{2}+\frac{1}{2} \Phi(q)$, that is, $q \leq \Phi^{-1}(1 / 3) \leq-0.4$ and

$$
\frac{1}{\sqrt{2 \pi}} p \exp \left(\frac{-p^{2}}{2}\right) \leq \Phi(p)-\frac{1}{2}=\frac{1}{2} \Phi(q)
$$

So, by Lemma 1 ,

$$
\begin{aligned}
4 p^{2} \exp \left(q^{2}-p^{2}\right) & \leq 2 \pi \Phi(q)^{2} \exp \left(q^{2}\right)=h(-q) \leq h(0.4) \\
& =2 \pi \Phi(-0.4)^{2} \exp (0.16) \leq 0.876<\ln 4 .
\end{aligned}
$$

CASE 2. $q^{2} \leq p^{2}$ and $q \leq 0$. Then $\Phi(q) \leq \frac{1}{2}$, so that $p \leq \Phi^{-1}(0.75) \leq 0.679$ and

$$
4 p^{2} \exp \left(q^{2}-p^{2}\right)-p^{2} \leq 3 p^{2}<\ln 4
$$

CASE 3. $q>0$. We will consider $p$ as a function of $q$. Then we have

$$
\frac{d}{d q}\left(p^{2}-q^{2}\right)=2 p \frac{d p}{d q}-2 q=p \exp \left(\left(p^{2}-q^{2}\right) / 2\right)-2 q .
$$

However, by Lemma $3, q \sqrt{h(q)}<p \sqrt{h(p)}$ so

$$
\frac{2 q}{p}<\frac{2 \sqrt{h(p)}}{\sqrt{h(q)}}=2 \exp \left(\frac{\left(p^{2}-q^{2}\right)}{2}\right) \frac{T(p)}{T(q)}=\exp \left(\left(p^{2}-q^{2}\right) / 2\right) .
$$

Thus $p^{2}-q^{2}$ is an increasing function of $q$. Moreover, by Lemma $1, h(q) \geq$ $h(p)$, hence $\exp ^{p^{2}-q^{2}} \leq 4$ and $p^{2}-q^{2} \leq \ln 4$. Let us consider Table 2 .

One can easily check that for $k=1,2,3$,

$$
T_{k} \geq T\left(q_{k}\right) \geq 2 T\left(p_{k}\right)
$$

and

$$
p_{k}^{2}-q_{k}^{2} \leq d_{k} \leq\left(2 \pi T_{k+1}^{2} \exp \left(q_{k+1}^{2}\right)\right)^{-1}-q_{k+1}^{2} \leq h\left(q_{k+1}\right)^{-1}-q_{k+1}^{2} .
$$

Suppose that $q \in\left[q_{k}, q_{k-1}\right)$ for some $k=1, \ldots, 4$, where additionally we put $q_{0}=\infty$. Then by Lemma 2 and monotonicity of $p^{2}-q^{2}$ we get for $k=2,3,4$,

$$
h(q)^{-1}-q^{2} \geq h\left(q_{k}\right)^{-1}-q_{k}^{2} \geq d_{k-1} \geq p_{k-1}^{2}-q_{k-1}^{2} \geq p^{2}-q^{2}
$$

TABLE 2

\begin{tabular}{ccccc}
\hline $\boldsymbol{k}$ & $\boldsymbol{q}_{\boldsymbol{k}}$ & $\boldsymbol{T}_{\boldsymbol{k}}$ & $\boldsymbol{p}_{\boldsymbol{k}}$ & $\boldsymbol{d}_{\boldsymbol{k}}$ \\
\hline 1 & 1.20 & 0.1152 & 1.58 & 1.057 \\
2 & 0.52 & 0.3016 & 1.04 & 0.812 \\
3 & 0.20 & 0.4208 & 0.81 & 0.617 \\
4 & 0.00 & 0.5000 & & \\
\hline
\end{tabular}


and for $k=1$,

$$
h(q)^{-1}-q^{2} \geq h\left(q_{1}\right)^{-1}-q_{1}^{2} \geq\left(2 \pi T_{1}^{2} \exp \left(q_{1}^{2}\right)\right)^{-1}-q_{1}^{2} \geq \ln 4 \geq p^{2}-q^{2} .
$$

Hence

$$
p^{2} h(q) \leq 1
$$

Moreover, by Lemma $4, F(q) \geq F(p)$, so

$$
h(p)^{-1}-h(q)^{-1} \leq 2 \ln (T(q) / T(p))=\ln 4 .
$$

Finally, we get

$$
4 \exp \left(q^{2}-p^{2}\right) p^{2}-p^{2}=p^{2}\left(\frac{h(q)}{h(p)}-1\right)=p^{2} h(q)\left(\frac{1}{h(p)}-\frac{1}{h(q)}\right) \leq 1 \ln 4
$$

CoROllary 4. If $w^{2}-p^{2} \geq \ln 4$ then

$$
w \gamma_{2}^{+}(A) \geq p \gamma_{2}^{+}(P)=\sqrt{\frac{2}{\pi}} p \exp \left(\frac{-p^{2}}{2}\right) .
$$

Proof. Suppose that $\gamma_{2}(A)=2 \Phi(p)-1=\Phi(q)$; then

$$
\frac{1}{2}(1-\Phi(q))=1-\Phi(p)
$$

and by isoperimetric inequality,

$$
\gamma_{2}^{+}(A) \geq \frac{1}{\sqrt{2 \pi}} \exp \left(\frac{-q^{2}}{2}\right) .
$$

Hence if $w \gamma_{2}^{+}(A)<p \gamma_{2}^{+}(P)$ then $w<2 p \exp \left(\left(q^{2}-p^{2}\right) / 2\right)$ so by Lemma 13,

$$
w^{2}-p^{2}<4 p^{2} \exp \left(q^{2}-p^{2}\right)-p^{2} \leq \ln 4
$$

and we get a contradiction.

Proof of Theorem 2. By Corollary 4 we may and will assume that

$$
w^{2}-p^{2}<\ln 4
$$

Let us define for $x \in[0, w)$,

$$
\begin{aligned}
& A(x)=\left\{\left(x_{1}, x_{2}\right) \in(-w, w) \times R:\left|x_{1}\right|<x \text { or } x_{2} \leq f\left(x_{1}\right)\right\}, \\
& \gamma(x)=\gamma_{2}(A(x))
\end{aligned}
$$

and

$$
d(x)=\frac{1}{2 \pi} \int_{x}^{w} \exp \left(\frac{-\left(t^{2}+f^{2}(t)\right)}{2}\right) \sqrt{1+\left(f^{\prime}(t)\right)^{2}} d t
$$

Let $a(x)$ and $g(x)$ be given by

$$
\Psi(a(x))=\gamma(x)
$$


and

$$
g(x)=2 \pi w d(x)+\sqrt{2 \pi} x(1-\Phi(y)) \exp \left(-x^{2} / 2\right)-\sqrt{2 \pi} a \exp \left(-a^{2} / 2\right),
$$

where $y=f(x)$. Then $A(0)=A, \gamma(0)=\gamma_{2}(A), 2 d(0)=\gamma_{2}^{+}(A)$ and $a(0)=p$, so in order to prove the theorem we have to show that $g(0) \geq 0$. Since $a(w)=w$ and $d(w)=0$ we have $g(w)=0$, so it is enough to show that $g$ is nonincreasing on $[0, w)$.

Let us also notice that for $y=f(x)$ and $a=a(x)$ we have

$$
\Phi(y) \Phi(w)+(1-\Phi(y)) \Phi(x) \geq \frac{1}{2}+\frac{1}{2} \gamma(x)=\Phi(a) .
$$

Moreover if $k=-f^{\prime}(x)$ then

$$
\begin{aligned}
& d^{\prime}(x)=-\frac{\sqrt{1+k^{2}}}{2 \pi} \exp \left(\frac{-\left(x^{2}+y^{2}\right)}{2}\right), \\
& \gamma^{\prime}(x)=2 \frac{1-\Phi(y)}{\sqrt{2 \pi}} \exp \left(\frac{-x^{2}}{2}\right) .
\end{aligned}
$$

So since $a^{\prime}(x) \Psi^{\prime}(a)=\gamma^{\prime}(x)$, we have

$$
a^{\prime}(x)=(1-\Phi(y)) \exp \left(\left(a^{2}-x^{2}\right) / 2\right) .
$$

So we get that

$$
\begin{aligned}
\exp \left(x^{2} / 2\right) g^{\prime}(x)= & \sqrt{2 \pi}\left(a^{2}-x^{2}\right)(1-\Phi(y))+k x \exp \left(-y^{2} / 2\right) \\
& -\sqrt{1+k^{2}} w \exp \left(-y^{2} / 2\right) .
\end{aligned}
$$

Therefore by Lemma 12 the proof will be complete if we establish the following claim.

Claim. Under the above notation it is not possible that $y=f(x)>1.5$, $a(x)^{2}>x^{2}+y^{2}+1.5$ and $w^{2}-p^{2}<\ln 4$.

Proof of THE Claim. Suppose that it is possible, so for some $0 \leq x<w$, we have $y=f(x)>1.5, a=a(x)>\sqrt{x^{2}+y^{2}+1.5}$ and $w^{2}<p^{2}+\ln 4$. Let the line $l$ tangent to the set $A$ in the point $(x, y)$ intersect the $y$-axis at the point $(0, s)$. Then since the set $A$ is convex it is contained in the half-plane below the line $l$. Therefore

$$
\gamma_{2}(D)+\Phi(u) \geq \frac{1}{2}+\frac{1}{2} \gamma_{2}(A)
$$

where $D$ is a set of points which have negative first coordinate and lie above the line $l$ and $u$ is a distance from the origin to $l$. As $\gamma_{2}(D) \leq \frac{1}{2}(1-\Phi(s))$ and $\gamma_{2}(A)=2 \Phi(p)-1$ we obtain

$$
1-\Phi(u) \leq 1-\Phi(p)+\frac{1}{2}(1-\Phi(s)) .
$$

So by Lemma 8 ,

$$
\frac{1}{2} \exp \left(\left(u^{2}-s^{2}\right) / 2\right)+\exp \left(\left(u^{2}-p^{2}\right) / 2\right) \geq 1 .
$$


In particular, since $u^{2} \leq s^{2}$ and $u^{2} \leq x^{2}+y^{2}$ we get that $w^{2} \leq p^{2}+\ln 4 \leq$ $u^{2}+2 \ln 4 \leq x^{2}+y^{2}+2 \ln 4$. Let us notice that by (15),

$$
(1-\Phi(y))(\Phi(a)-\Phi(x)) \leq \Phi(y)(\Phi(w)-\Phi(a)) .
$$

Since $a^{2}>x^{2}+y^{2}+1.5>x^{2}+3.75$, by Lemma 7 , we obtain

$\Phi(a)-\Phi(x) \geq(1-\Phi(x))\left(1-\exp \left(\left(x^{2}-a^{2}\right) / 2\right)\right) \geq(1-\Phi(x))(1-\exp (-1.875))$.

Moreover (see the proof of Lemma 7),

$$
\Phi(w)-\Phi(a) \leq \frac{1}{\sqrt{2 \pi} a}\left(\exp \left(\frac{-a^{2}}{2}\right)-\exp \left(\frac{-w^{2}}{2}\right)\right)
$$

and by Lemma 2,

$$
1-\Phi(y) \geq \frac{1}{\sqrt{2 \pi\left(y^{2}+2\right)}} \exp \left(\frac{-y^{2}}{2}\right)
$$

Hence

$$
\begin{aligned}
&(1-\Phi(x)) \exp \left(\frac{x^{2}}{2}\right) \\
& \leq(1-\exp (-1.875))^{-1} \frac{\sqrt{y^{2}+2}}{a} \\
& \quad \times\left(\exp \left(\frac{x^{2}+y^{2}-a^{2}}{2}\right)-\exp \left(\frac{x^{2}+y^{2}-w^{2}}{2}\right)\right) .
\end{aligned}
$$

Suppose first that $x \leq 0.8$; then by Lemma 1 ,

$$
(1-\Phi(x)) \exp \left(x^{2} / 2\right) \geq(1-\Phi(0.8)) \exp (0.32) \geq 0.29 \text {. }
$$

On the other hand, since $\sqrt{y^{2}+2} / a \leq \sqrt{\left(y^{2}+2\right) /\left(y^{2}+1.5\right)} \leq \sqrt{4.25 / 3.75}$ we get by (17) that

$$
(1-\Phi(x)) \exp \left(\frac{x^{2}}{2}\right) \leq(1-\exp (-1.875))^{-1} \sqrt{\frac{4.25}{3.75}}\left(\exp (-0.75)-\frac{1}{4}\right) \leq 0.28
$$

This contradiction shows that $x>0.8$ and then $a \geq \sqrt{x^{2}+y^{2}+1.5} \geq \sqrt{y^{2}+2}$. Thus by (17),

$$
\begin{aligned}
(1- & \Phi(x)) \exp \left(\frac{x^{2}}{2}\right) \\
\leq & (1-\exp (-1.875))^{-1} \\
& \times\left(\exp \left(\left(x^{2}+y^{2}-a^{2}\right) / 2\right)-\exp \left(\left(x^{2}+y^{2}-w^{2}\right) / 2\right)\right) .
\end{aligned}
$$


TABLE 3

\begin{tabular}{ccccc}
\hline $\boldsymbol{k}$ & $\boldsymbol{d}_{\boldsymbol{k}}$ & $\boldsymbol{x}_{\boldsymbol{k}}$ & $\boldsymbol{T}_{\boldsymbol{k}}$ & $\boldsymbol{c}_{\boldsymbol{k}}$ \\
\hline 1 & 1.50 & 3.23 & 0.0005 & 0.092 \\
2 & 1.85 & 2.29 & 0.0109 & 0.149 \\
3 & 2.12 & 1.80 & 0.0358 & 0.180 \\
4 & 2.27 & 1.53 & 0.0629 & 0.202 \\
5 & 2.39 & 1.31 & 0.0950 & 0.224 \\
6 & 2.52 & 1.05 & 0.1468 & 0.254 \\
7 & 2.71 & 0.51 & & \\
\hline
\end{tabular}

Let us consider Table 3 . The reader may check that the numbers in the table satisfy the following inequalities for $k=1, \ldots, 6$ :

$$
\begin{gathered}
T_{k} \leq 1-\Phi\left(x_{k}\right), \quad c_{k}<T_{k} \exp \left(x_{k}^{2} / 2\right) \\
c_{k}>(1-\exp (-1.875))^{-1}\left(\exp (-0.75)-\exp \left(-d_{k+1} / 2\right)\right)
\end{gathered}
$$

and

$$
x_{k} \geq \sqrt{2 \ln 4-d_{k}}+\sqrt{-2 \ln \left(2-4 \exp \left(-d_{k} / 2\right)\right)} .
$$

The last inequality holds also for $k=7$. Suppose that

$$
w^{2}-x^{2}-y^{2} \in\left[d_{k}, d_{k+1}\right] \text { for some } k=1,2, \ldots, 7,
$$

where we additionally define $d_{8}=\infty$. Then

$$
x^{2}+y^{2}-u^{2}=x^{2}+y^{2}-w^{2}+w^{2}-u^{2} \leq-d_{k}+2 \ln 4
$$

and

$$
u^{2}-p^{2} \leq x^{2}+y^{2}-p^{2} \leq x^{2}+y^{2}-w^{2}+\ln 4 \leq \ln 4-d_{k} .
$$

Thus by (16) we get

$$
s^{2}-u^{2} \leq-2 \ln \left(2-2 \exp \left(\left(u^{2}-p^{2}\right) / 2\right)\right) \leq-2 \ln \left(2-4 \exp \left(-d_{k} / 2\right)\right) .
$$

Consider the triangle $A B C$ with $A=(0,0), B=(x, y)$ and $C=(0, s)$; then by the Pythagoras theorem,

$$
\begin{aligned}
x & \leq|B C| \leq \sqrt{|A C|^{2}-u^{2}}+\sqrt{|A B|^{2}-u^{2}} \\
& \leq \sqrt{-2 \ln \left(2-4 \exp \left(-d_{k} / 2\right)\right)}+\sqrt{2 \ln 4-d_{k}} \leq x_{k} .
\end{aligned}
$$

Hence if $k=7, x<0.8$ which contradicts our previous assumption. For $k<7$ we have by Lemma 1 ,

$$
(1-\Phi(x)) \exp \left(x^{2} / 2\right) \geq\left(1-\Phi\left(x_{k}\right)\right) \exp \left(x_{k}^{2} / 2\right)>c_{k}
$$


and

$$
\begin{gathered}
(1-\exp (-1.875))^{-1}\left(\exp \left(\left(x^{2}+y^{2}-a^{2}\right) / 2\right)-\exp \left(\left(x^{2}+y^{2}-w^{2}\right) / 2\right)\right) \\
\leq(1-\exp (-1.875))^{-1}\left(\exp (-0.75)-\exp \left(-d_{k+1} / 2\right)\right)<c_{k}
\end{gathered}
$$

The above inequalities contradict (18) and the proof is now complete.

Acknowledgments. This work was done while the first-named author was visiting Southeast Applied Analysis Center at School of Mathematics, Georgia Institute of Technology. The research of the second-named author was performed at and partially supported by the Feinberg Graduate School of the Weizmann Institute of Science in Rehovot, Israel.

\title{
REFERENCES
}

[1] Ehrhard, A. (1983). Symetrisation dans l'espace de Gauss. Math. Scand. 53 281-301.

[2] Hitczenko, P., Kwapień, S., Li, W., Schechtman, G., Schlumprecht, T. and Zinn, J. (1998). Hypercontractivity and comparison of moments of iterated maxima and minima of independent random variables. Electron. J. Probab. 3.

[3] ITô K. and McKean H. P. (1965). Diffusion Processes and Their Sample Paths. Springer, New York.

[4] KWAPIEŃ, S. and SAWA, J. (1993). On some conjecture concerning Gaussian measures of dilatations of convex symmetric sets. Studia Math. 105 173-187.

[5] Sudakov, V. N. and ZalgalleR, V. A. (1974). Some problems on centrally symmetric convex bodies. In Problems in Global Geometry. Zap. Naučn. Sem. Leningrad. Otdel. Mat. Inst. Steklov. (LOMI) 45 75-82, 119 (in Russian).

[6] SzAREK, S. (1991). Condition numbers of random matrices. J. Complexity 7 131-149.

\author{
INSTITUTE OF MATHEMATICS \\ WARSAW UNIVERSITY \\ BANACHA 2 \\ 02-097 WARSZAWA \\ POLAND \\ E-MAIL: rlatala@mimuw.edu.pl \\ koles@mimuw.edu.pl
}

